Pacific Journal of Mathematics

FIXED-POINT-FREE OPERATOR GROUPS OF ORDER 8 


\section{FIXED-POINT-FREE OPERATOR GROUPS OF ORDER 8}

\section{Fletcher Gross}

Let $A$ be a group of order $2^{n}$ which acts as a fixed-pointfree group of operators on the finite solvable group $G$. If no additional assumptions are made concerning $G$, then "reasonable" upper bounds on the nilpotent length, $l(G)$, of $G$ have been obtained only when $A$ is cyclic [Gross] or elementary abelian [Shult]. As a small step in extending the class of 2-groups $A$ for which such bounds exist, it is shown in the present paper that if $|A|=8$, then $l(G) \leqq 3$ if $A$ is elementary abelian or quaternion and $l(G) \leqq 4$ otherwise.

Unfortunately, the author was unable to generalize his methods of proof to a wider class of groups.

The notation used in this paper agrees with that of [1] with two additions: (1) If $G$ is a linear group operating on $V$ and $U$ is a $G$-invariant subspace, then $\{G \mid U\}$ denotes the restriction of $G$ to $U$; and (2) $F_{0}(G)=1$ and $F_{n+1}(G) / F_{n}(G)$ is the greatest normal nilpotent subgroup of $G / F_{n}(G)$.

THeorem 1. Let $G=N Q$ be a finite solvable linear group over a field $K$ whose characteristic is not 2 and does not divide $\left|F_{1}(N)\right|$. Assume that $N$ is a normal 2-complement of $G$ and $Q$ is a group of order 8 containing an element $x$ of order 4 . If, in addition, $C_{N}(Q)=1$ and $\sum_{g \in Q} g=0$, then it must must follow that

$$
\left[x^{2}, F_{2}(N) / F_{1}(N)\right]=1 \text {. }
$$

Proof. According to the hypothesis $Q$ can be any group of order 8 except an elementary abelian group. If $Q$ is cyclic, this theorem is a special case of [4, Th. 1.2], and if $Q$ is a quaternion group, then a stronger result is possible. Thus the main interest in the theorem is when $Q$ is either dihedral or is the direct product of cyclic groups of orders 4 and 2.

To prove the theorem we first notice that extending $K$ affects neither hypothesis or conclusion. Thus we may as well assume that $K$ is algebraically closed. We now assure that $G$ is a minimal counterexample to the theorem and let $V$ be the space on which $G$ operates.

Choose $S$ to be a subgroup of $F_{2}(N)$ such that $Q$ normalizes $S$, $\left[x^{2}, S\right] \not F_{1}(N)$, and $S$ is minimal with respect to the above properties. $S$ must be a $p$-group for some prime $p$. Now $Q$ normalizes $\left[x^{2}, S\right]$, and $\left[x^{2},\left[x^{2}, S\right]\right]=\left[x^{2}, S\right][2]$. Due to the minimality of $S$, this implies that $\left[x^{2}, S\right]=S$. 
Now $C_{S}\left(O_{p},\left(F_{1}(N)\right)\right)=S \cap F_{1}(N)$. Thus there is an $r$-group $R$ for some prime $r \neq p$ such that $Q S$ normalizes $R, R \leqq F_{1}(N),[S, R] \neq 1$, and $R$ is minimal with respect to the above properties. $R$ must be a special $r$-group, and $R / R^{\prime}$ must be transformed irreducibly by $Q S$.

Since the characteristic of $K$ does not divide $\left|F_{1}(N)\right|, V$ is a completely reducible $K-R$ module. From this and the fact that $[S, R] \triangleleft Q S R$, it follows that $V$ contains a maximal $K-Q S R$ submodule $M$ such that $[S, R]$ is not the identity on $V / M$. Now let $H$ be the kernel of the representation of $Q S R$ afforded by $V / M$.

Since $\langle x\rangle$ must be faithfully represented on $V / M$, we have that either $Q \cap H=1$ or $Q / Q \cap H$ is cyclic of order 4 . But $Q$ has no nonzero fixed vector in $V$ and so certainly has none in $V / M$. Thus if $Q / Q \cap H$ is cyclic of order 4 , then it follows from [4] that $\left[x^{2}, S, R\right]=1$. Hence we must have $Q \cap H=1$. This implies that $Q S R / H$ acting as a linear group on $V / M$ satisfies the hypothesis but not the conclusion of the theorem. Therefore, in proving the theorem we may as well assume that $G=Q S R$ and that $V$ is an irreducible $K-G$ module.

Clifford's theorem now implies that $V$ is a completely reducible $K-S R$ module and $V=V_{1} \oplus V_{2} \oplus \cdots \oplus V_{t}$ where the $V_{i}$ are the homogeneous $K-S R$ modules. $Q$ must permute the $V_{i}$ transitively, and, since $[S, R] \triangleleft Q S R$, it must be that $\left\{[S, R] \mid V_{i}\right\} \neq 1$ for all $i$.

We now proceed to prove that $t=1$, or, in other words, that $V$ is a homogeneous $K-S R$ module. For this purpose let

$$
Q_{i}=\left\{g \mid g \in Q, V_{i} g=V_{i}\right\}
$$

and

$$
C_{i}=\left\{g \mid g \in Q_{i},\left\{[g, S R] \mid V_{i}\right\}=1\right\} \text {. }
$$

Then $Q_{i}$ and $Q_{j}$ as well as $C_{i}$ and $C_{j}$ are conjugate in $Q$ for all $i$ and $j$. $\left[Q: Q_{i}\right]=t, V_{i}$ is an irreducible $K-Q_{i} S R$ module, and $\left\{\sum_{g \in Q_{i}} g \mid V_{i}\right\}=0$ for all $i$. The last fact implies that $Q_{i} \neq 1$. Since $\left\{\left[x^{2}, S\right] \mid V_{i}\right\} \neq 1$, $x^{2}$ cannot belong to $C_{i}$.

\section{Lemma. $\quad C_{i}=1$ for all $i$.}

Proof. Suppose $C_{i} \neq 1$. Since $\langle x\rangle \cap C_{i}=1$, it follows that $C_{i}$ is cyclic of order 2 generated by an element $y_{i}$. Now $C_{R S}(x)$ is normalized by $Q$. It follows from this and the fact that conjugation by $x$ transitively permutes the $y_{i}$ that $\left[u, y_{i}\right]=\left[u, y_{j}\right]$ for all $i$ and $j$ and all $u \in C_{R S}(x)$. Since $\left[u, y_{i}\right]$ is represented by the identity on $V_{i}$, this all implies that $\left[C_{R S}(x), y_{i}\right]=1$ for all $i$. Since $x$ and $y_{i}$ generate $Q$, we obtain that $C_{R S}(x)=C_{R S}(Q)=1$. Hence $x$ acts as a fixed-point-free automorphism on $R S$. From this follows $\left[x^{2}, S, R\right]=1$ [3] which is a contradiction. 
LEMma. $Q_{i}=Q$ and $t=1$.

Proof. If $Q_{i}$ is elementary abelian, it follows from [7, Th. 4.1] that $C_{i} \neq 1$. Thus, since $Q_{i} \neq 1$, we must have either $Q_{i}=Q$ or $Q_{i}$ is cyclic of order 4 generated by an element $y$. If $Q_{i}$ is cyclic of order 4 we must have $y^{2}=x^{2}$ because $Q$ only has 8 elements. Now $Q_{i}$ can have no nonzero fixed vector in $V_{i}$. Theorem 1.2 of [4] now yields that $\left[x^{2}, S, R\right]$ is represented by the identity on $V_{i}$. Since this is impossible, $Q_{i}$ must be $Q$. Then $t=\left[Q: Q_{i}\right]=1$ and so $V$ is a homogeneous $K-S R$ module.

\section{Corollary. $\quad Z(S R)=R^{\prime}=1$.}

Proof. $Z(S R)$ is represented by scalar matrices and so $Q$ must centralize $Z(S R)$. Thus $Z(S R) \leqq C_{R S}(Q)=1$. Now $R^{\prime}$ is normalized by $Q S$ and so, due to the minimality of $R$, we must have $\left[S, R^{\prime}\right]=1$. Therefore $R^{\prime} \leqq Z(S R)$.

Now let $V=U_{1} \oplus U_{2} \oplus \cdots \oplus U_{s}$ where the $U_{i}$ are the homogeneous $K-R$ submodules of $V$. Let $H_{i}=\left\{g \mid g \in Q S, U_{i} g=U_{i}\right\}$ and $S_{i}=$ $H_{i} \cap S$. Now $S Q$ must permute the $U_{i}$ transitively since $V$ is an irreducible $K-Q S R$ module. Thus $s=\left[Q S: H_{i}\right]$ for all $i$. But $V$ is a homogeneous $K-S R$ module. This implies that $\left(U_{i} S\right) Q=U_{i} S$. Hence $U_{i} S=V$ for all $i$. Therefore $s=\left[S: S_{i}\right]=\left[Q S: H_{i}\right]$ which means that $H_{i}$ must contain a Sylow 2-subgroup of $S Q$. Since the $H_{i}$ are all conjugate in $Q S$, this implies that $Q \leqq H_{i}$ for some $i, i=1$ say. Then $Q$ fixes $U_{1}$. Let $R_{1}$ be the kernel of the representation of $R$ afforded by $U_{1}$. Clearly $R_{1}$ is normalized by $Q$. But $R$ is abelian and so $R$ is represented by scalar matrices on $U_{1}$. It now follows that $\left[R / R_{1}, Q\right]=1$. Since $C_{R}(Q)=1$, this implies that $R_{1}=R$. But, since $V$ is an irreducible $K-Q S R$ module and $R \triangleleft Q S R$, this is impossible. This contradiction proves the theorem.

THEOREM 2. Let $G=N Q$ be a finite solvable linear group over a field $K$ whose characteristic does not divide $\left|F_{1}(N)\right|$. Assume that $N$ is a normal 2-complement of $G$ and $Q$ is an ordinary quaternion group. If, in addition, $C_{\mathrm{V}}(Q)=1$ and $\sum_{g \in Q} g=0$, then it must follow that $\left[Q^{\prime}, F_{1}(N)\right]=1$.

Proof. Extending $K$ affects neither hypothesis nor conclusion. Thus we assume that $K$ is algebraically closed. If $\left[Q^{\prime}, F_{1}(N)\right] \neq 1$, then there is a subgroup $P$ of $F_{1}(N)$ such that $Q$ normalizes $P, Q^{\prime}$ does not centralize $P$, and $P$ is minimal with respect to the above properties. Then $P$ is a special $p$-group for some prime $p$ and $P / P^{\prime}$ is transformed faithfully and irreducibly by $Q$. This implies that 
$\left|P / P^{\prime}\right|=p^{2}$, and so $P$ is either elementary abelian of order $p^{2}$ or extraspecial of order $p^{3}$ and exponent $p$.

If $V$ is the vector space on which $G$ operates, then

$$
V=V_{1} \oplus V_{2} \oplus \cdots
$$

where the $V_{i}$ are the homogeneous $K-P$ modules. By renumbering, we may assume that $\left[Q^{\prime}, P\right]$ is not the identity on $V_{1}$. Now if $Q$, as a permutation group on the $V_{i}$, had an orbit of length 8 , then $\sum_{g \in Q} g$ would not be 0 . This implies that $Q^{\prime}$ must fix $V_{1}$.

If $\left\{P \mid V_{1}\right\}$ is abelian, then $P$ is represented by scalar matrices on $V_{1}$ and so we would have $\left\{\left[Q^{\prime}, P\right] \mid V_{1}\right\}=1$. Thus $\left\{P \mid V_{1}\right\}$ is not abelian. This implies that $P=\left\{P \mid V_{1}\right\}=$ an extra-special $p$-group of order $p^{3}$ and exponent $p$.

Now let $H=\left\{g \mid g \in Q, V_{1} g=V_{1}\right\}$. In order that $\sum_{g \in Q} g=0$, we must have $\left\{\sum_{g \in I I} g \mid V_{1}\right\}=0$. Now a faithful irreducible $K$-representation of $P$ is uniquely determined by the representation of $P^{\prime}$ [6]. It follows from this that $H=C_{Q}\left(P^{\prime}\right)$. Since $C_{P}(Q)=1, H \neq P$. But the automorphism group of $P^{\prime}$ is cyclic. Thus $Q / H$ is cyclic. This implies that $H$ is cyclic of order 4 . Let $x$ generate $H$ and let $y$ be an element of $Q$ not contained in $H$.

Case 1. $p \equiv 1(\bmod 4)$.

Suppose first that char $(K) \neq 2$. Then Theorem 3.1 of [7] implies that $\left\{\left[x^{2}, P\right] \mid V_{1}\right\}=1$, which is a contradiction. If char $(K)=2$, then Theorem $B$ of [6] leads to $\left\{x^{3}+x^{2}+x+1 \mid V_{1}\right\} \neq 0$, also a contradiction.

Case 2. $p \equiv 3(\bmod 4)$.

In this case $G F(p)$ does not contain a primitive 4 th root of unity. Since $Q$ faithfully transforms $P / P^{\prime}$, it follows that there elements $a$, $b$ generating $P$ such that

$$
a^{y} \equiv b, b^{y} \equiv a^{-1}\left(\bmod P^{\prime}\right) .
$$

But this implies that $[a, b]^{y}=\left[b, a^{-1}\right]=[a, b]$, contrary to $y \in C_{Q}\left(P^{\prime}\right)$.

Theorem 3. Let $Q$ be a group of order 8 which acts as a fixedpoint-free group of automorphisms of the finite group G. Then $G$ is solvable and $l(G) \leqq 3$ if $Q$ is either elementary abelian or $a$ quaternion group and $l(G) \leqq 4$ otherwise. The upper bound in the case when $Q$ is elementary abelian or a quaternion group is bestpossible.

Proof. If $G$ admits a 2-group as a fixed-point-free operator group, 
then $G$ must have odd order and so $G$ must be solvable from the Feit-Thompson Theorem [1]. If $Q$ is elementary abelian, the result follows from Thorem 4.3 of [7]. Therefore assume that $Q$ has an elemen $x$ of order 4 . We now use induction on $|G|$.

If $H_{1}, H_{2}$ are distinct minimal $Q$-admissible normal subgroups, then $l(G) \leqq l\left[(G / H) \times\left(G / H_{2}\right)\right]=\operatorname{Max}\left\{l\left(G / H_{1}\right), l\left(G / H_{2}\right)\right\}$. Thus in proving the theorem we may assume that $G$ has only one minimal $Q$-admissible normal subgroup. Hence $F_{1}(G)$ is a $p$-group for some prime $p$. Now let $N=G / F_{1}(G)$ and consider $N Q$ as a linear group acting on $V$ where $V$ is $F_{1}(G) / D\left(F_{1}(G)\right)$ written additively. Theorems 1 and 2 imply that $\left[x^{2}, F_{k}(N) / F_{k-1}(N)\right]=1$ where $k=1$ if $Q$ is a quaternion group and $k=2$ otherwise. It follows from this that $\left[x^{2}, N / F_{k-1}(N)\right]=1$. But then $N / F_{k-1}(N)$ admits a fixed-point-free operator group of order 4. This implies that $l\left(N / F_{k-1}(N)\right) \leqq 2$. We now have that

$$
l(G)=1+l(N)=1+(k-1)+l\left(N / F_{k-1}(N)\right) \leqq k+2 .
$$

Finally, the claim of best-possible in the statement of the theorem is justified by [5].

\section{REFERENCES}

1. W. Feit and J. Thompson, Solvability of groups of odd order, Pacific J. Math. 13 (1963), 775-1029.

2. G. Glauberman, Fixed points in groups with operator groups, Math. Z. 84 (1964), 120-125.

3. D. Gorenstein and I. Herstein, Finite groups admitting a fixed-point-free automorphism of order 4, Amer. J. Math. 83 (1961), 71-78.

4. F. Gross, Groups admitting a fixed-point-free automorphism of order $2^{n}$, Pacific J. Math. (in press)

5. - A note on fixed-point-free solvable operator groups, Proc. Amer. Math. Soc. (in press).

6. P. Hall and G. Higman, On the p-length of p-soluble groups and reduction theorems for Burnside's problem, Proc. London Math. Soc. (3) 6 (1956), 1-42.

7. E. Shult, On groups admitting fixed-point-free abelian groups, Illinois J. Math. 9 (1965), 701-720.

Received March 19, 1968.

UNIVERSITY OF UTAH

Salt Lake City, Utah 



\section{PACIFIC JOURNAL OF MATHEMATICS}

\section{EDITORS}

H. ROYDEN

Stanford University

Stanford, California

\section{R. $R$ PHELPS}

University of Washington

Seattle, Washington 98105

\section{J. DugunduI}

Department of Mathematics

University of Southern California

Los Angeles, California 90007

RICHARD ARENS

University of California

Los Angeles, California 90024

\section{ASSOCIATE EDITORS}

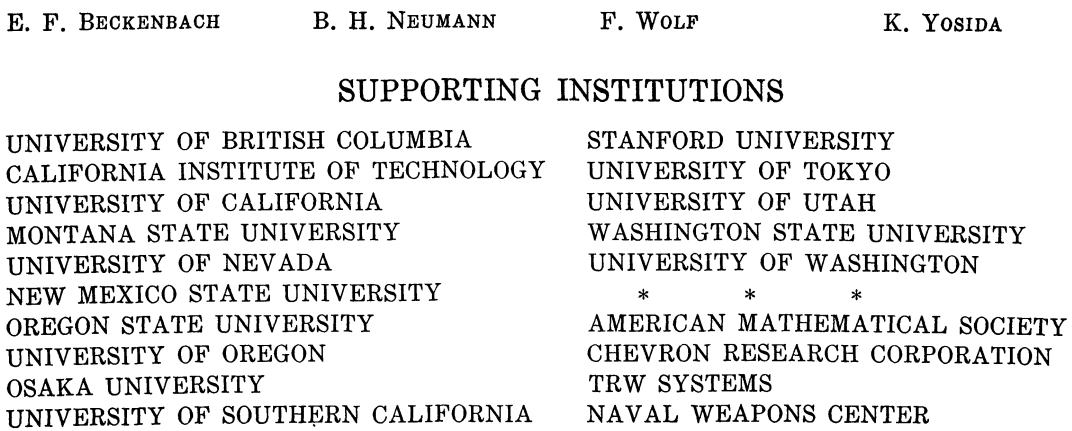

The Supporting Institutions listed above contribute to the cost of publication of this Journal, but they are not owners or publishers and have no responsibility for its content or policies.

Mathematical papers intended for publication in the Pacific Journal of Mathematics should be in typed form or offset-reproduced, double spaced with large margins. Underline Greek letters in red, German in green, and script in blue. The first paragraph or two must be capable of being used separately as a synopsis of the entire paper. It should not contain references to the bibliography. Manuscripts, in duplicate if possible, may be sent to any one of the four editors. Please classify according to the scheme of Math. Rev. 36, 1539-1546. All other communications to the editors should be addressed to the managing editor, Richard Arens, University of California, Los Angeles, California, 90024.

50 reprints are provided free for each article; additional copies may be obtained at cost in multiples of 50 .

The Pacific Journal of Mathematics is published monthly. Effective with Volume 16 the price per volume (3 numbers) is $\$ 8.00$; single issues, $\$ 3.00$. Special price for current issues to individual faculty members of supporting institutions and to individual members of the American Mathematical Society: $\$ 4.00$ per volume; single issues $\$ 1.50$. Back numbers are available.

Subscriptions, orders for back numbers, and changes of address should be sent to Pacific Journal of Mathematics, 103 Highland Boulevard, Berkeley, California, 94708.

PUBLISHED BY PACIFIC JOURNAL OF MATHEMATICS, A NON-PROFIT CORPORATION

Printed at Kokusai Bunken Insatsusha (International Academic Printing Co., Ltd.), 7-17, Fujimi 2-chome, Chiyoda-ku, Tokyo, Japan. 


\section{Pacific Journal of Mathematics}

Vol. 28, No. $2 \quad$ April, 1969

Richard Arens and Donald George Babbitt, The geometry of relativistic

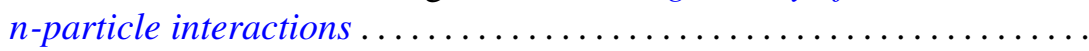

Kirby Alan Baker, Hypotopological spaces and their embeddings in lattices

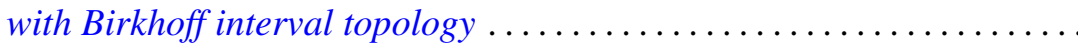

J. Lennart (John) Berggren, Finite groups in which every element is conjugate to its inverse ........................... 289

Beverly L. Brechner, Homeomorphism groups of dendrons . . . . . . . . . . . 295

Robert Ray Colby and Edgar Andrews Rutter, QF - 3 rings with zero singular ideal ................................. 303

Stephen Daniel Comer, Classes without the amalgamation property....... 309

Stephen D. Fisher, Bounded approximation by rational functions ......... 319

Robert Gaines, Continuous dependence for two-point boundary value problems..................................... 327

Bernard Russel Gelbaum, Banach algebra bundles ............... 337

Moses Glasner and Richard Emanuel Katz, Function-theoretic degeneracy criteria for Riemannian manifolds ...................... 351

Fletcher Gross, Fixed-point-free operator groups of order $8 \ldots \ldots \ldots \ldots 357$

Sav Roman Harasymiv, On approximation by dilations of distributions . . . . 363

Cheong Seng Hoo, Nilpotency class of a map and Stasheff's criterion ... . . 375

Richard Emanuel Katz, A note on extremal length and modutus.......... 381

H. L. Krall and I. M. Sheffer, Difference equations for some orthogonal polynomials ................................

Yu-Lee Lee, On the construction of lower radical properties ........... 393

Robert Phillips, Liouville's theorem........................... 397

Yum-Tong Siu, Analytic sheaf cohomology groups of dimension $n$ of

n-dimensional noncompact complex manifolds ..... . .

Michael Samuel Skaff, Vector valued Orlicz spaces. II...

James DeWitt Stein, Homomorphisms of $B^{*}$-algebras .... . .

Mark Lawrence Teply, Torsionfree injective modules .... . . .

Richard R. Tucker, The $\delta^{2}$-process and related topics. II .

David William Walkup and Roger Jean-Baptiste Robert Wets, Lifting

projections of convex polyhedra...

Thomas Paul Whaley, Large sublattices of a lattice. 